DISCUSSION

\title{
A synthetic soft rock for laboratory model studies
}

\author{
I. W. JOHNSTON and S. K. CHOI (1986). Geotechnique 36, No. 2, 251-263
}

Professor J. D. Sage and Professor R. A. D'An-
drea, Worcester Polytechnic Institute
The Writers agree that, by testing a homogencous isotropic substance whose engineering behaviour resembles that of intact natural rock, the number of variables which influence the scatter of test results can be reduced. However, since geologic discontinuities generally govern the in situ behaviour of natural rock, it is often difficult to predict field behaviour confidently from laboratory results of tests performed on intact specimens. In certain situations, such as the prediction of shear resistance along a naturally occurring joint surface, it would be advantageous to reproduce the irregularities present along the field joint surface on the artificial material which is eventually tested in the laboratory. Fig. 15 of the Paper portrays irregular surfaces that are similar to joint profiles used in the Writers' studies and the Writers would like details concerning the ease in casting 'Johnstone' specimens in the field.

Furthermore the Writers would be interested in any results of shear testing along the surfaces shown in fig. 15 . The Writers are currently engaged in a study of sliding along fabricated rock-like surfaces to examine the nature of the load-deformation characteristics of jointed rock. To replicate the characteristics of jointed crystalline rocks, a commercially available dental cement known as 'Die Keen' is being used to cast specimens. Die Keen is essentially calcium sulphate with a proprietary additive supplement which is manufactured by Columbus Dental Supply in St Louis, Missouri. The powdered Die Kcen is casily mixed with water and cast in a mould. After drying at room temperature the resultant material exhibits a linear stress-strain relationship and brittle behaviour under zero confining stress; detailed properties are listed in Table 1.

Results of non-dilatant direct shear tests performed by the Writers indicate that the ability of the synthetic rock to resist sliding along a fabricated joint plane is governed by the tensile strength of the rock. Specimens whose geometry resembles that shown in fig. 15(a) undergo tensile fracture along the back of the asperities when tested under low levels of normal stress. Tensile failure was presumed from the roughness of the fractured surface and supported by finite element analysis of the joint surface assuming a linear elastic material. This is in contrast with a MohrCoulomb failure criterion first proposed by Patton (1966) and supported by others. The Writers are attempting to determine whether this tension-controlled behaviour is due to the geometry of the simulated joint, the brittle nature of the material tested, the imposed zero dilatancy or a combination of factors. Any comments from the Authors regarding shear across surfaces of their soft rock would be useful.

\section{REFERENCE}

Patton, F. D. (1966). Multiple modes of shear failure in rock. Proc. Ist Congr. International Society of Rock Mechanics, Lisbon 1, 509-513.

\section{Authors' reply}

As pointed out by the Writers, the in situ behaviour of natural rock masses is generally governed by the presence of geological discontinuities and therefore the extrapolation of laboratory test results on intact rock to describe the performance of natural rock masses must be treated with caution. The Authors agree wholeheartedly with this generally expressed opinion. However, the Authors also believe that a rational and numerate understanding of natural rock mass behaviour, particularly for soft rocks, can only be achieved through a comprehensive appreciation of the mechanisms of the individual components involved in this behaviour. By gradually developing more complex, and therefore more realistic, numerical models of the load-deformation responses of intact rock and of discontinuities, it may be possible eventually to combine these models, to introduce natural variability and to make rational predictions of mass behaviour. Attempts to predict the complex mass behaviour without a detailed understanding of the individual components can only be achieved by empirical, and therefore subjective, methods which have, so far, dominated methods of prediction in rock engineering.

As deduced by the Writers from fig. 15 of the Paper, the synthetic soft rock has also been used to investigate the shear behaviour of rough rock 
Table 1. Average properties of room temperature cured Die Keen

\begin{tabular}{ll}
\hline Mix & $21 \mathrm{~cm}^{3}$ of water to $100 \mathrm{~g}$ of Die Keen \\
Unconfined compressive strength & $46.2 \mathrm{MPa}$ \\
Secant modulus at zero confining stress & $38200 \mathrm{MPa}$ \\
Poisson's ratio & 0.3 \\
Tensile strength (via ASTM D2936) & $4.9 \mathrm{MPa}$ \\
\hline
\end{tabular}

joints. Although these investigations have been specifically related to the rough joint formed between the shaft of a socketed pile and the parent rock mass, the results obtained are also relevant to the general problem of the loaddeformation of rock joints in shear. In response to the Writers' interest in the results of these shear tests, many hundreds of these tests have been conducted on not only synthetic rocks but also natural rock samples and plaster-based samples. It would be impractical to present even a summary of these results in this discussion and therefore the Writers are referred to Johnston \& Lam (1984), Lam (1983), Lam \& Johnston (1982), Williams (1980) and Lam \& Johnston (1985a). A wide range of previously unpublished data, principally concerning the behaviour of random surface geometry as illustrated in fig. 15(b) of the Paper, will be submitted for possible publication.

The Writers report that, in their test programme, the response of rough joint surfaces to applied shear loading is governed by the tensile strength of their synthetic rock. While there is some evidence of local tensile failure in the tests reported by Lam (1983) and Lam \& Johnston (1985a) on the synthetic Johnstone (and by Williams (1980) and Johnston, Lam \& Williams (1987) on natural mudstone), the principal mechanism leading to failure is a combination of sliding along asperity faces followed by either the crushing of asperities or the shearing of asperities. Therefore, for Johnstone, tensile strength is not a major influence. However, in a series of tests carried out by Williams (1980) on samples made of plaster, there was evidence that tensile mechanisms may be more important. When comparing the properties of the plaster with those of Johnstone, the plaster was considerably stronger, had a greater voids ratio and was considerably more brittle. It is also likely that the internal structure of the plaster was quite different from that of Johnstone. Therefore, since the Die Keen is even stronger and perhaps more brittle than the plaster, it is reasonable to expect that the principal mechanism of failure may be as suggested by the Writers and governed by tensile strength.

The principal reason for the development of Johnstone was to obtain a reasonable representative model for the naturally occurring soft rock of concern to the Authors. For reasons discussed in the Paper, plaster-based model materials cast in moulds without compression were considered unsuitable. Although materials such as Die Keen may be convenient for modelling stronger rocks, it should be remembered that strength is only one parameter and that a complete modelling material should respond with the same mechanisms as the rock it is purported to model. The Writers do not provide information concerning the internal structure of Dic Kecn, but if its voids ratio is relatively high and typical of cast materials such as plaster compared with the very low voids ratios that are typical of rocks of uniaxial compressive strength of about $50 \mathrm{MPa}$ then it is likely that substantial differences may exist in the respective mechanisms of behaviour. This is the reason why a substantial compressive stress is used in the manufacture of Johnstone.

The Writers also request details concerning the ease of casting Johnstone in the field. All casting operations are carried out in the laboratory. Samples with representative surface roughness profiles are manufactured using compression moulds consisting of base plates which have been machined to form dies for the profile required. These profiles are based on the roughness characteristics measured by the techniques discussed in Lam \& Johnston (1985b).

\section{REFERENCES}

Johnston, I. W. \& Lam, T. S. K. (1984). Frictional characteristics of planar concrete-rock interfaces under constant normal stiffness conditions. Proc. 4th Aust.-N.Z. Conf. Geomech., Perth 2, 397-401.

Johnston, I. W., Lam, T. S. K. \& Williams, A. F. (1987). Constant normal stiffness direct shear testing for socketed pile design in weak rock. Géotechnique 37, No. 1, 83-89.

Lam, T. S. K. (1983). Shear behaviour of concrete rock joints. PhD thesis, Monash University.

Lam, T. S. K. \& Johnston, I. W. (1982). A constant normal stiffness direct shear machine. Proc. 7 th S. E. Asian Geotech. Conf., Hong Kong 1, 805-820.

Lam, T. S. K. \& Johnston, I. W. (1985a). Shear behaviour of regular concrete-rock joints-evaluation. Departmental Report, Department of Civil Engineering, Monash University.

Lam, T. S. K. \& Johnston, I. W. (1985b). A scanning device to quantify joint surface roughness. Geotech. Test. J. 8, 117-124.

Williams, A. F. (1980). The design and performance of piles socketed into weak rock. PhD thesis, Monash University. 